\title{
Clinical Options in Relapsed or Refractory Hodgkin Lymphoma: An Updated Review
}

\author{
Roberta Fedele, ${ }^{1}$ Massimo Martino, ${ }^{1}$ Anna Grazia Recchia, ${ }^{2}$ Giuseppe Irrera, \\ Massimo Gentile, ${ }^{3}$ and Fortunato Morabito ${ }^{2,3}$ \\ ${ }^{1}$ Hematology and Stem Cell Transplant Unit, Azienda Ospedaliera BMM, 89100 Reggio Calabria, Italy \\ ${ }^{2}$ Biotechnology Research Unit, Azienda Sanitaria Provinciale di Cosenza, 87051 Aprigliano, Italy \\ ${ }^{3}$ Hematology Unit, Azienda Ospedaliera di Cosenza, 87100 Cosenza, Italy
}

Correspondence should be addressed to Roberta Fedele; r.fedele@yahoo.it

Received 18 July 2015; Accepted 22 October 2015

Academic Editor: Daniel Olive

Copyright (C) 2015 Roberta Fedele et al. This is an open access article distributed under the Creative Commons Attribution License, which permits unrestricted use, distribution, and reproduction in any medium, provided the original work is properly cited.

\begin{abstract}
Hodgkin lymphoma (HL) is a potentially curable lymphoma, and modern therapy is expected to successfully cure more than $80 \%$ of the patients. Second-line salvage high-dose chemotherapy and autologous stem cell transplantation (auto-SCT) have an established role in the management of refractory and relapsed HL, leading to long-lasting responses in approximately $50 \%$ of relapsed patients and a minority of refractory patients. Patients progressing after intensive treatments, such as auto-SCT, have a very poor outcome. Allogeneic SCT represents the only strategy with a curative potential for these patients; however, its role is controversial. Based on recent knowledge of HL pathology, biology, and immunology, antibody-drug conjugates targeting CD30, small molecule inhibitors of cell signaling, and antibodies that inhibit immune checkpoints are currently explored. This review will discuss the clinical results regarding auto-SCT and allo-SCT as well as the current role of emerging new treatment strategies.
\end{abstract}

\section{Introduction}

Hodgkin lymphoma (HL) is a potentially curable lymphoma with distinct histology, biological behavior, and clinical characteristics. Thomas Hodgkin first described the disorder in 1832. In the 20th century, with the realization that the disease consisted of a lymphoid malignancy, it was renamed HL. It is a relatively rare disease and accounts for approximately $10 \%$ of all malignant lymphomas, with about 9,200 estimated new cases and 1,200 estimated deaths per year in the United States [1]. The treatment of HL has evolved over the past three decades, and modern therapy is expected to successfully cure over $80 \%$ of patients [2]. Second-line salvage highdose chemotherapy (HDC) and autologous stem cell transplantation (auto-SCT) have become the standard care for refractory/relapsed HL, leading to long-lasting responses in approximately $50 \%$ of relapsed patients and in a minority of refractory patients [3]. Disease recurrence or progression after auto-SCT is associated with very poor prognosis [4] and patients have an estimated average survival of less than 3 years [5]. However, because HL is a rare cancer that is highly curable, the development of new drugs for the treatment of HL has been very slow [6]. With growing knowledge of HL pathology, biology, and immunology, several therapeutic targets have been identified and are currently under preclinical and clinical investigation [7]. The aim of drug development in $\mathrm{HL}$ is not only to cure patients, but also to go further and decrease the toxic effects of therapy.

In this review, we summarize the most recent updates on the management of patients with relapsed or refractory HL and the role of novel therapeutic approaches. We also discuss the role of consolidation strategies such as HDC and autoSCT and reduced-intensity (RIC) allogeneic stem cell transplantation (allo-SCT).

\section{Autologous Stem Cell Transplantation}

According to retrospective and prospective as well as randomized studies, HDC followed by auto-SCT can rescue $30 \%$ to $80 \%$ of relapsed/refractory HL patients [8-14]. 
In the BNLI trial [12], relapsed patients were treated with conventional dose mini-BEAM (carmustine, etoposide, cytarabine, and melphalan) or high-dose BEAM with autoSCT. Both event-free survival (EFS) and progression-free survival (PFS) showed significant differences in favor of BEAM plus transplant ( $p=0.025$ and $p=0.005$, resp.). In the GHSG trial [13], patients who relapsed after chemotherapy were randomly given four courses of mini-BEAM+dexamethasone (dexa-mini-BEAM) or two courses of dexa-mini-BEAM followed by BEAM and auto-SCT. Freedom from treatment failure (FFTF) in 3 years was significantly better for patients given BEAM and auto-SCT (55\%) than for those on dexamini-BEAM (34\%; $p=0.019)$. Overall survival (OS) of patients given either treatment did not differ significantly. Recently, the GHSG group [14] evaluated the impact of sequential HDC before myeloablative therapy. Patients with histologically confirmed, relapsed HL were treated with two cycles of dexamethasone, cytarabine, and cisplatin, and those without disease progression were then randomly divided between standard and experimental treatment arms. In the standard arm, patients received myeloablative therapy with BEAM followed by auto-SCT. In the experimental arm, patients received sequential cyclophosphamide, methotrexate, and etoposide in high doses before BEAM. Mortality was similar in both arms (20\% and $18 \%)$. With a median observation time of 42 months, there was no significant difference in terms of FFTF $(p=0.56)$ and OS $(p=0.82)$ between arms. FFTF in 3 years was $62 \%$ and OS was $80 \%$. Results demonstrated that sequential HDC did not improve outcome and was associated with more adverse events and toxicity. Based on the data presented, the authors concluded that two cycles of intensified conventional chemotherapy (DHAP) followed by HDC (BEAM) and auto-SCT are an effective and safe treatment strategy for patients with relapsed HL.

On the basis of this study, BEAM is considered the gold standard conditioning regimen for auto-SCT. However, due to drug constraints of carmustine, this drug is often replaced by a variety of agents, including fotemustine [15], bendamustine [16], and thiotepa [17].

Sweetenham et al. [18] published a retrospective analysis of 175 patients with HL who did not undergo remission after induction therapy and results were reported to the European Group for Bone Marrow Transplantation (EBMT). The 5year actuarial OS and PFS rates were $36 \%$ and $32 \%$, respectively, and results were very similar to those reported from single-institution series and from the Autologous Blood and Marrow Transplant Registry (ABMTR) [19]. The ABMTR series includes 122 patients with HL who have never achieved remission. The definition of failure to achieve remission differs from that in the EBMT series, in that it includes only those patients who had a documented disease progression or tissue confirmation of persistent disease in residual radiographic abnormalities. With a median follow-up of 28 months from the date of auto-SCT, the 3-year actuarial PFS and OS rates in this series were $38 \%$ and $50 \%$, respectively. The GELTAMO Cooperative Group [20] presented the results of 62 patients treated with an auto-SCT for refractory HL. One-year transplant-related mortality (TRM) was $14 \%$. The response rate in 3 months after auto-SCT was 52\%. Actuarial 5 -year time to treatment failure (TTF) and OS were $15 \%$ and $26 \%$, respectively. The presence of B symptoms at auto-SCT was the only adverse prognostic factor significantly influencing TTF. The presence of B symptoms at diagnosis, MOPPlike regimens as first-line therapy, bulky disease at auto-SCT, and two or more lines of therapy before auto-SCT adversely influenced OS.

Tandem auto-SCT for HL has been evaluated in a small number of studies [21-24] and in the most recent guidelines from the American Society for Blood and Marrow Transplantation it is not recommended, although further studies may be warranted in high-risk patients [25].

\section{Allogeneic Stem Cell Transplantation}

Although there is relatively limited accessible data regarding the best approach for patients who relapse after an autoSCT, the available information supports the benefit of alloSCT versus standard therapy [25-28]. Evidence of a graft versus HL (GVHL) effect comes from the demonstration that the development of graft versus host disease (GVHD) after allo-SCT is associated with a lower relapse rate $[29,30]$. Moreover, the most direct evidence for a graft versus malignancy effect comes from the disease responses to donor lymphocyte infusions (DLIs). Peggs et al. [31] assessed the impact of DLI on relapse incidence when administered for mixed chimerism and the utility of DLI as salvage therapy when given for relapse in 76 consecutive patients with multiple relapsed or refractory HL, who underwent allo-SCT that incorporated in vivo T-cell depletion. The results demonstrated the potential for allogeneic immunotherapy with DLIs both to reduce relapse risk and to induce durable antitumor responses.

Despite early data showing promisingly low relapse rates after allo-SCT, the transplantation community was not very enthusiastic about considering allo-SCT for HL patients, because of the exceedingly high nonrelapse mortality (NRM). Registry data $[32,33]$ has shown that allo-SCT after myeloablative conditioning results in lower relapse rates but significantly higher toxicity than auto-SCT. Although the poor results after myeloablative conditioning can be explained by the very poor risk features of heavily pretreated patients included in these early trials, high TRM has been associated with high incidence of GVHD and infections after transplantation. Results of allo-SCT can be reasonably optimized with a better patient selection and the use of targeted and less toxic therapies to achieve an adequate response for patients.

In the last years, the use of RIC has reduced NRM and improved OS [34] and the percentage of patients with refractory and relapsed HL treated using this approach has been growing steadily in Europe [35]. Robinson et al. [36] conducted a retrospective analysis of 285 patients with HL who underwent a RIC allo-SCT in order to identify prognostic factors of outcome. Eighty percent of patients had undergone a prior auto-SCT and 25\% had refractory disease at transplant. NRM was associated with chemorefractory disease, poor performance status, age $>45$, and transplantation before 2002. For patients with no risk factors, the 3-year 
NRM rate was $12.5 \%$ compared to $46.2 \%$ for patients with two or more risk factors. The use of an unrelated donor had no adverse effects on the NRM. The development of chronic GVHD was associated with a lower relapse rate. The disease progression rate in 1 and 5 years was $41 \%$ and $58.7 \%$, respectively, and was associated with chemorefractory disease and extent of prior therapy. PFS and OS were both associated with performance status and disease status at transplant. Patients with neither risk factor had a 3-year PFS and OS of $42 \%$ and $56 \%$, respectively, compared to $8 \%$ and $25 \%$ for patients with one or more risk factors. Relapse within 6 months of a prior auto-SCT was associated with a higher relapse rate and a lower PFS.

In the analysis by Robinson et al., the authors also identified important clinical parameters predicting transplant outcomes. RIC allo-SCT may be an effective salvage strategy for the minority of patients with good risk features who relapse after an auto-SCT, with similar outcomes for both sibling and matched unrelated donor (MUD) transplants. On the other hand, for patients with chemorefractory disease or a poor performance status, the overall outcome is poor and it is difficult to recommend RIC allo-SCT for these patients.

Burroughs et al. [37] evaluated the outcome of RIC allo-SCT for patients with relapsed or refractory HL based on different donor cell sources. Ninety patients with HL were treated with nonmyeloablative conditioning followed by allo-SCT from HLA-matched related, unrelated, or HLAhaploidentical related donors. The nonmyeloablative preparative regimen consisted in either 2-Gy total body irradiation (TBI) or combination with fludarabine $30 \mathrm{mg} / \mathrm{m} 2 /$ day followed by postgrafting immunosuppression with mycophenolate mofetil or cyclosporine/tacrolimus. Patients were heavily pretreated with a median of five regimens and most patients had failed auto-SCT and local radiation therapy. With a median follow-up of 25 months, the 2-year OS, the PFS, and incidence of relapsed/progressive disease were $53 \%, 23 \%$, and $56 \%$ (HLA-matched related); 58\%, 29\%, and 63\% (unrelated); and $58 \%, 51 \%$, and $40 \%$ (HLA-haploidentical related), respectively. NRM was significantly lower for HLA-haploidentical related $(p=0.02)$ recipients compared to HLA-matched related recipients. There were promising results with significantly decreased risks of relapse for HLA-haploidentical related recipients compared to HLA-matched related ( $p=$ $0.01)$ and unrelated $(p=0.03)$ recipients. The incidence of acute GVHD grade III/IV and extensive chronic GVHD was $16 \% / 50 \%$ (HLA-matched related), $8 \% / 63 \%$ (unrelated), and $11 \% / 35 \%$ (HLA-haploidentical related), respectively.

Raiola et al. [38] confirmed in 26 advanced HL patients the results published by the Baltimore/Seattle group [39], using haplo-mismatched marrow grafts and posttransplantation cyclophosphamide. The procedure was feasible, with a low rate of GVHD and NRM, and was associated with a durable remission in a high proportion of patients. The 4-year OS and EFS were $77 \%$ and $63 \%$, respectively. EFS was statistically different when patients were stratified according to disease phase: 1 -year PFS was $100 \%, 67 \%$, and $37 \%$ for patients in complete remission (CR) $(n=9)$, partial remission (PR) $(n=9)$, or resistant disease $(n=8)$, respectively $(p=0.02)$. Actuarial survival was not statistically different in the three groups $(p=0.1)$. The cumulative incidence of NRM was $4 \%$. The 100-day cumulative incidence of grade I and grade II-IV acute GVHD was $4 \%$ and $24 \%$, respectively; the cumulative 3-year incidence of moderate chronic GVHD was $9 \%$.

The Lymphoma Working Party (LWP) of the EBMT, together with the GEL/TAMO [40], undertook the largest multicenter phase II prospective clinical trial presented up to now with the objective of analyzing the NRM and other major outcome parameters after allo-SCT in relapsed/refractory HL. In this study, 92 patients with an HLA-identical sibling, a MUD, or a one antigen mismatched, unrelated donor were treated with salvage chemotherapy followed by RIC alloSCT. Fludarabine $(150 \mathrm{mg} / \mathrm{m} 2$ intravenously) and melphalan $(140 \mathrm{mg} / \mathrm{m} 2$ intravenously) were used as the conditioning regimen. The addition of antithymocyte globulin was used as GVHD prophylaxis for recipients of grafts from unrelated donors. The NRM rate was $8 \%$ in 100 days and $15 \%$ in 1 year. Relapse was the major cause of failure. The PFS rate was $48 \%$ in 1 year and $24 \%$ in 4 years. The OS rate was $71 \%$ in 1 year and $43 \%$ in 4 years. The results of this study emphasize the role of RIC allo-SCT in patients with relapsed/refractory HL after auto-SCT. The plateau phase in the survival curve of the subset of patients allografted in CR indicates the existence of a clinically beneficial GVHL effect. Chronic GVHD was associated with a significantly lower relapse incidence after transplantation and consequently a significant improvement of PFS.

Recently, the LWP of the EBMT has reported [41] the results on the outcome of the second allo-SCT (allo-SCT2) performed in one hundred and forty patients with lymphoma, of which $31 \%$ were affected by HL. Three-year PFS, OS, relapse incidence, and NRM were 19\%, 29\%, 58\%, and $23 \%$, respectively. PFS and OS were significantly affected by refractory disease at allo-SCT- 2 and by a short interval between allo-SCT-1 and allo-SCT-2. Long-term PFS was observed in particular in patients with HL, T-cell lymphoma, and indolent lymphoma where a GVHL effect was assumed [42]. In fact, considering that, in many patients, chronic GVHD was absent after allo-SCT-1 but not after allo-SCT-2, it is possible to conclude that the second allotransplant might induce an effective allo-response in patients in which GVHD failed to appear after the first transplant. Allo-SCT-2 can result in long-term disease control in patients with lymphoma recurrence after allo-SCT-1, in particular if relapse occurs late and is chemosensitive.

\section{Brentuximab Vedotin}

The expression of CD30 by Reed-Sternberg cells (RSc) coupled with its highly restricted expression makes it an obvious target for monoclonal antibody therapy $[43,44]$. Results from two clinical studies using first-generation naked anti-CD30 monoclonal antibodies in patients with relapsed HL have been disappointing, perhaps reflecting their poor antigen binding and/or effector cell activation properties $[45,46]$. In an alternate strategy, the anti-CD30 antibody cAC10 was conjugated to a synthetic antimicrotubule agent, monomethyl auristatin E (MMAE), resulting in the novel immunotoxin conjugate brentuximab vedotin [47]. In a 
phase I dose escalation trial that enrolled 45 patients with relapsed or refractory $\mathrm{CD} 30$ + hematologic malignancies [48], objective responses, including 11 CRs, were observed in 17 patients and tumor regression was observed in $86 \%$ of evaluable patients. Seventy-three percent of patients in that trial had undergone auto-SCT. Brentuximab vedotin $(1.8 \mathrm{mg} / \mathrm{kg}$ intravenously every 3 weeks) was subsequently evaluated in a pivotal phase 2 study of 102 patients with relapsed/refractory CD30+ HL after auto-SCT [49]. Objective responses were documented in $75 \%$ of patients, with CRs observed in $34 \%$ of patients, as determined by an independent radiology review facility. The estimated 12 -month survival rate was $89 \%$ and the median PFS was 5.6 months. Adverse events associated with brentuximab vedotin were typically of grade I/II and were treated through standard supportive care. Cumulative peripheral neuropathy, the most meaningful clinical adverse effect, improved or resolved completely in $80 \%$ of patients during the study.

Median OS and PFS were estimated in 40.5 months and 9.3 months, respectively. Improved outcomes were observed in patients who achieved a CR on brentuximab vedotin, with estimated 3-year OS and PFS rates of $73 \%$ and $58 \%$, respectively, in this group of patients [50]. Of the 34 patients who obtained CR, 16 (47\%) remain progression-free after a median of 53.3 months (range, 29.0 to 56.2 months); 12 patients remain progression-free without a consolidative allo-SCT. Younger age, good performance status, and lower disease burden at baseline were characteristic of patients who achieved a CR and were favorable prognostic factors for OS.

On the basis of these studies, brentuximab vedotin has been approved for the treatment of adult patients with relapsed or refractory CD30+ HL following auto-SCT or following at least two prior therapies with auto-SCT or multiagent chemotherapy.

The randomized, double-blind, placebo-controlled, phase 3 AETHERA study [51] demonstrated that brentuximab vedotin improves PFS when given as early consolidation after auto-SCT in patients with HL with risk factors for relapse or progression after transplantation. The high risk of progression after auto-SCT is defined by the presence of primary refractory HL (failure to achieve CR), relapsed HL with an initial remission duration of less than 12 months, or extranodal involvement at the start of pretransplantation salvage chemotherapy. Compared with historical survival data for high-risk patients with HL undergoing auto-SCT, the 3 -year OS rate exceeding $80 \%$ in this study is remarkable.

A recent SIE, SIES, GITMO position paper declares that there is now evidence for recommending brentuximab vedotin also in HL patients refractory to salvage chemotherapy who are auto-SCT candidates and as a consolidation strategy after auto-SCT. The use of brentuximab vedotin in HL after relapse from allo-SCT or as first-line therapy is at present only experimental [52]. The Expert Panel recommends that, in the approved indications of brentuximab vedotin treatment for HL, treatment evaluation must be performed after 4 courses, and the subsequent treatment should be determined according to the response. In patients with $\mathrm{HL}$ attaining a CR, either an early consolidation program including allo-SCT or brentuximab vedotin therapy continued up to 16 cycles is the approved indications. It is necessary to perform clinical trials to clarify which one of the two strategies is more appropriate. Early allo-SCT should strongly be considered in patients with HL attaining a PR. Patients not eligible for transplant should be treated with brentuximab vedotin up to a maximum of 16 cycles. In patients with HL and a stable disease, the decision to continue brentuximab vedotin should rely on a patient-centered balance between clinical benefits and risks. In patients with HL and a progressive disease, brentuximab vedotin therapy should be discontinued and patients must be enrolled in clinical trials.

\section{Bendamustine}

Bendamustine is a bifunctional alkylating agent with only partial cross-resistance to other alkylating drugs, making it an attractive agent for use in the relapsed setting [53]. Although it was developed in the 1960s and used in Germany for both HL and non-HL, it has been approved for treatment of chronic lymphatic leukemia and indolent B-cell non-HL [54] and limited data exist regarding its activity in HL patients. Moskowitz et al. [55] performed a phase II study evaluating the efficacy and toxicity of bendamustine in relapsed and refractory HL. Thirty-six patients were enrolled, and 25 patients were potentially eligible for allo-SCT. Bendamustine $120 \mathrm{mg} / \mathrm{m} 2$ was administered on days 1 and 2 of each 28 -day cycle for a total of six cycles of treatment. The dose of bendamustine was reduced to $100 \mathrm{mg} / \mathrm{m} 2$ for treatment delays $>5$ days because of neutropenia or thrombocytopenia. The dose was further reduced to $70 \mathrm{mg} / \mathrm{m} 2$ for subsequent delays of $>5$ days for neutropenia or thrombocytopenia. The most common nonhematologic toxicities were fatigue (primarily grade I) and nausea (primarily grade I). Thrombocytopenia was the most common hematologic toxicity, with $20 \%$ of patients experiencing grade III or IV thrombocytopenia. The overall response rate (ORR) for the 36 patients was 53\%, demonstrating that bendamustine is a good option for heavily treated patients with relapsed and refractory HL who could proceed to consolidative SCT. Zinzani et al. [56] reported two cases of patients relapsed/refractory after brentuximab vedotin were successfully treated with bendamustine indicating that patients with HL relapsed/refractory to brentuximab vedotin therapy may be chemosensitive and may obtain a good response to subsequent bendamustine treatment. Zinzani et al. [57], after these case reports, performed a retrospective study on 27 heavily pretreated patients with relapsed or refractory HL, who had all received brentuximab vedotin as their last treatment and who showed disease progression, refractory disease, or early relapse, when retreated with bendamustine. The ORR was $55.5 \%$, with 10 of 27 patients $(37.0 \%)$ obtaining a CR. In comparison, the ORR previously observed with brentuximab vedotin in the same subset of patients was much lower (18.5\%).

Considering the promising results of brentuximab vedotin and bendamustine as single drugs on patients with relapsed/refractory HL and their independent mechanisms of action with manageable safety profiles, a phase I-II study was performed evaluating the safety and efficacy of brentuximab vedotin in combination with bendamustine 
for the treatment of patients with HL first relapse [58]. Brentuximab vedotin $1.8 \mathrm{mg} / \mathrm{kg}$ on day 1 in combination with bendamustine $90 \mathrm{mg} / \mathrm{m} 2$ on days 1 and 2 of 3-week-cycles for up to 6 cycles had a manageable safety profile with premedication. The CR rate of the combination was $82 \%$ and ORR 94\%. The majority of CRs (24/28 patients) were documented after 2 cycles of combination therapy and, in these patients, stem cell mobilization and collection were performed with success. These data indicate a promising approach for maximizing responses prior SCT in relapsed/ refractory HL patients after frontline therapy. Promising data were reported by O'Connor and colleagues [59] on the combination of brentuximab vedotin and bendamustine in relapsed/refractory HL and anaplastic large T-cell lymphoma.

\section{Panobinostat and Mocetinostat}

Agents that target acetylases may regulate several oncogenic pathways including cell cycle progression, cell survival, angiogenesis, and antitumor immunity. Panobinostat and mocetinostat target histone deacetylase (HDAC) and these agents may be effective in patients with HL by modulating serum cytokine levels and the expression of PD-1 on intratumoral T-cells.

Based on promising results from a phase I study that included 13 patients with relapsed HL [60], a large pivotal international phase II study was initiated. Oral panobinostat was administered at a dose of $40 \mathrm{mg}$ three times per week, every week, in 21-day cycles. Dose delays and modifications for management of adverse events were permitted, but the lowest dose allowed on study was $20 \mathrm{mg}$. Efficacy was evaluated every 2 cycles by imaging studies. Surprisingly, patients were enrolled in less than one year. The median age was 32 years (range, 18-75), and the median number of prior chemotherapeutic regimens was 4 (range, 1-7). Importantly, the median time to relapse after the first auto-SCT was only 8 months, which represents a poor prognostic indicator. Moreover, 37\% of the patients did not respond to their last prior therapy. Twelve patients also received prior allo-SCT.

In a phase II study, 129 patients with relapsed and refractory HL received $40 \mathrm{mg}$ of panobinostat orally three times per week [61]. Treatment with panobinostat was effective as tumor reductions were seen in $74 \%$ of patients, and ORs were achieved by 35 patients (27\%). Thirty patients (23\%) had partial responses to treatment and five patients (4\%) had CRs. The median duration of response was 6.9 months, and the median PFS was 6.1 months. The treatment was reasonably well tolerated with common drug-related grade I/II adverse effects as diarrhea, nausea, fatigue, vomiting, and anorexia. Common drug-related grade III/IV adverse events were thrombocytopenia, anemia, and neutropenia. The thrombocytopenia was manageable and reversible with dose hold and modification.

Considering the synergistic activity of HDAC inhibitors with other therapies $[62,63]$, association studies of HDAC inhibitors combined with chemotherapy, monoclonal antibodies, and small molecule inhibitors will be evaluated. A phase I study of panobinostat combined with lenalidomide in relapsed HL is ongoing [64].
The safety and efficacy of mocetinostat were recently evaluated in a phase II study in 51 patients with relapsed classical HL [65]. Mocetinostat was given orally 3 times per week ( $85 \mathrm{mg}$ to $110 \mathrm{mg}$ starting doses) for 1 year in the absence of disease progression or prohibitive toxicity. Initially, 23 patients were enrolled in the $110 \mathrm{mg}$ cohort. Subsequently, because toxicity-related dose reductions were necessary in the $110 \mathrm{mg}$ cohort, 28 additional patients were treated with a dose of $85 \mathrm{mg}$. The disease control rate was 35\% (eight of 23 patients) in the $110 \mathrm{mg}$ group and 25\% (seven of $28)$ in the $85 \mathrm{mg}$ group. Three of the $10(30 \%)$ patients in the $85 \mathrm{mg}$ group achieved partial remissions. Furthermore, grade III and IV toxicity (mainly fatigue, with no significant hematologic toxicity) was reduced to $20 \%$. Overall, $80 \%$ of the 30 evaluable patients had some decrease in their tumor sizes. These data demonstrate that mocetinostat has a promising single-agent clinical activity with manageable toxicity in patients with relapsed classical HL.

\section{Everolimus}

The phosphatidylinositol 3-kinase/mammalian target of rapamycin (PI3K/mTOR) signaling pathway is one of the most aberrantly activated survival pathways in cancer, making it an important target for drug development [66]. Everolimus is an oral antineoplastic agent that targets this pathway, specifically the mTORcomplexl (mTORC1) that has been shown to be activated in patients with HL. Everolimus not only may target the signaling pathways within the RSc but may also suppress signaling within the immune infiltrate and production of cytokines present in the tumor microenvironment [67]. Nineteen evaluable patients with relapsed HL were treated with daily doses of $10 \mathrm{mg}$ everolimus, the ORR rate was $47 \%$, and 8 patients achieved PR and 1 CR [68]. The median time to disease progression was 7.2 months. The majority of patients had received multiple previous lines of therapy and $84 \%$ of the patients had undergone a previous auto-SCT. Grade III adverse events included thrombocytopenia and anemia. Considering that several signal transduction pathways are critical for the proliferation and survival of neoplastic Hodgkin RSc, including NF- $\kappa$ B, JAK-STAT, PI3K$\mathrm{AkT}$, and ERK [69], a combination of therapeutic approaches capable of targeting RSc along with reactive cells of the microenvironment might prolong the response duration of mTOR inhibitors to overcome chemorefractoriness.

\section{JAK Inhibitors}

The Janus kinase (JAK) and signal transducer and activator of transcription (STAT) pathway is an active mediator of cytokine signaling in the pathogenesis of solid and hematologic malignancies. The seven-member STAT family is composed of latent cytoplasmic transcription factors that are activated by phosphorylation intertwined in a network with activation that ultimately leads to cell proliferation. Aberrant activation of the JAK-STAT pathway has been demonstrated in patients with large granular lymphocytic leukemia, aplastic anemia, myelodysplastic syndrome, myeloproliferative disorders, and HL [70]. Pacritinib is an inhibitor of JAK2 
kinase with preclinical activity in a variety of hematological malignancies [71]. This JAK inhibitor has been used in a phase I clinical trial in patients with relapsed or refractory Hodgkin or non-Hodgkin lymphoma of any type except Burkitt or central nervous system lymphoma [72]. Doses of 100 to $600 \mathrm{mg} /$ day were tested, and treatment was well tolerated, with mostly grade I/II toxicities. Among the 34 patients' study, the ORR was $14 \%$, including three partial remissions. In the group of patients with HL, however, none of the 14 patients had a partial remission or better. However, at least five of the patients with HL did benefit from the treatment, with a decrease in the sites of active disease.

\section{Rituximab}

Rituximab has shown activity in nodular lymphocyte predominant HL. It is active in relapsed/refractory classical $\mathrm{HL}$ regardless of subtype or degree of CD20 expression on RS cells. Rationale of using rituximab in classic HL includes elimination of CD20+ reactive B-cells supporting RS cells, hence depriving malignant cells of survival signals and potentially increasing host immune responses [73]. In a pilot study [74], 22 patients with recurrent, classic HL who had received a minimum of two prior treatment regimens, regardless of whether H/RS cells expressed CD20, were treated with 6 weekly doses of $375 \mathrm{mg} / \mathrm{m} 2$ rituximab to selectively deplete infiltrating benign B-cells. Five patients (22\%) achieved partial or complete remission that lasted for a median of 7.8 months (range, 3.3-14.9 months). Remissions were observed in patients only at lymph node and splenic sites, but not at extranodal sites, and were irrespective of CD20 expression by H/RS cells. Furthermore, systemic (B) symptoms resolved in six of seven patients after therapy. These data need to be confirmed in clinical trial.

\section{Lenalidomide}

Lenalidomide is an immunomodulatory agent with several mechanisms of action, including direct induction of apoptosis in tumor cells, antiangiogenic effects, and the modulation of immune cells, such as natural killer cells and T-cells [75]. Limited data suggest that lenalidomide has clinical activity in relapsed/refractory HL. Fehniger et al. [76] evaluated 38 relapsed HL patients with $25 \mathrm{mg}$ /day of lenalidomide on days 1-21 of 28-day cycles; 33 of 38 patients had prior SCTs. The ORR to lenalidomide in the 35 evaluable patients was $17 \%$, with one CR. Additional six patients had stable disease (SD) lasting $>6$ months, resulting in an overall cytostatic response rate $(\mathrm{CR}+\mathrm{PR}+\mathrm{SD}>6$ months $)$ of $34 \%$. Treatment continued until progressive disease or an unacceptable adverse event. Kuruvilla et al. [77] evaluated lenalidomide in 14 patients with relapsed or refractory HL. Two patients achieved a PR (14\%), with additional seven patients having SD (50\%). The median time to progression in that study was only 3.2 months, with a median OS time of 9.1 months. Böll and colleagues used lenalidomide in 42 patients [78]. Preliminary results involving the first 24 patients have been reported. Twelve patients (50\%) had an objective response (11 with a PR and one with a CR), with additional eight patients achieving SD.
Further studies must be made to evaluate the actual efficacy and long-lasting effect of lenalidomide.

Lenalidomide was further evaluated in HL by the GHSG in a first-line phase I combination trial for older patients [79]. The GHSG aimed to improve the ABVD regimen by replacing bleomycin with lenalidomide (AVD-Rev) to improve both efficacy and tolerability of the regimen. Patients received four to eight cycles of AVD-Rev (standard-dose AVD on days 1 and 15 of a 28 -day cycle and lenalidomide daily from days 1 to 21 ) followed by radiotherapy. The daily lenalidomide dose for the first patient was $5 \mathrm{mg}$; maximum dose in this dose escalation trial was $25 \mathrm{mg}$. Twenty-five patients with a median age of 67 were enrolled. Sixty-eight had advanced stage disease, and $80 \%$ had B symptoms at diagnosis. After dose-limiting toxicity evaluation of 20 patients, a prespecified stopping criterion was reached and the recommended dose for a phase II trial was $25 \mathrm{mg}$. At least one grade III/IV toxicity occurred in all 22 patients who were treated at dose levels 20 and $25 \mathrm{mg}$, and 16 of those patients had a grade IV toxicity. The 1-year estimates for PFS and OS were 69 and $91 \%$, respectively. In summary, AVD-Rev displayed high efficacy and a manageable toxicity profile in older patients with HL and should be further evaluated in phase II/III trials. In addition, a phase II trial combining lenalidomide and panobinostat in patients with relapsed or refractory HL is currently recruiting.

\section{Anti-PD-1 Antibodies}

The concept that the immune system plays a critical role in controlling and eradicating cancer and that the immune response, driven by T-lymphocytes, is closely regulated through a complicated and delicate balance of inhibitory checkpoints and activating signals is well established [80, 81]. Programmed death-1 (PD-1) is one of the main immune checkpoint receptors that, when binding its programmed death-ligand-1 (PD-L1), determines the downregulation of the T-cell effector functions, thus contributing to the maintenance of the tolerance to tumor cells. The blockade of this pathway by anti-PD-1 and anti-PD-L1 antibodies may prevent this downregulation and allows T-cells to maintain their antitumor property and ability to mediate the tumor cell death [82-84]. The genes encoding the PD-1 ligands, PD-L1 and PD-L2, are key targets of chromosome 9p24.1 amplification, a recurrent genetic abnormality in the nodular sclerosis type of HL. The 9p24.1 amplicon also includes JAK2, and gene dose-dependent JAK-STAT activity further induces PD-1 ligand transcription [85]. The complementary mechanisms of PD-1 ligand overexpression in HL suggest that this disease may have genetically determined vulnerability to PD-1 blockade. For these reasons, in a phase I study, 23 extensively pretreated patients with relapsed or refractory $\mathrm{HL}$ were given every 2 weeks $3 \mathrm{mg} / \mathrm{kg}$ nivolumab, a fully human monoclonal IgG4 antibody directed against PD-1 [86]. The majority of these patients had previously received an autoSCT, and most had received previous brentuximab vedotin. Drug-related adverse events of any grade were reported in $18(78 \%)$ of 23 patients, and grade III drug-related adverse events were reported in five (22\%) patients. Of 23 patients, 
TABLE 1: Novel agents evaluated in relapsed/refractory HL patients after auto-SCT.

\begin{tabular}{|c|c|c|c|c|c|}
\hline Author & $\begin{array}{c}\text { Therapeutic agent(s), study } \\
\text { design }\end{array}$ & Pts. N1 & Pts. N2 & Response rate & $\begin{array}{l}\text { Median duration of } \\
\text { response }\end{array}$ \\
\hline Younes et al., 2010 [48] & Brentuximab vedotin, phase I & 42 & 33 & $\mathrm{ORR}=38 \% \mathrm{CR}=24 \%$ & 9.7 months \\
\hline Younes et al., 2012 [49] & Brentuximab vedotin, phase II & 102 & 102 & $\mathrm{ORR}=75 \% \mathrm{CR}=34 \%$ & $\begin{array}{l}20.5 \text { months for patients } \\
\text { in CR }\end{array}$ \\
\hline $\begin{array}{l}\text { Moskowitz et al., } 2013 \\
\text { [55] }\end{array}$ & Bendamustine, phase II & 35 & 27 & $\mathrm{ORR}=53 \% \mathrm{CR}=33 \%$ & 5 months \\
\hline Zinzani et al., 2015 [57] & Bendamustine, retrospective & 27 & 27 & $\mathrm{ORR}=55.5 \% \mathrm{CR}=37 \%$ & 8 months \\
\hline LaCasce et al., 2014 [58] & $\begin{array}{c}\text { Bendamustine }+ \text { brentuximab, } \\
\text { phases I-II }\end{array}$ & 45 & - & $\mathrm{ORR}=94 \% \mathrm{CR}=82 \%$ & NR \\
\hline Younes et al., 2012 [61] & Panobinostat, phase II & 129 & 129 & $\mathrm{ORR}=27 \% \mathrm{CR}=4 \%$ & 6.9 months \\
\hline Younes et al., 2011 [65] & Mocetinostat, phase II & 51 & 43 & $\mathrm{ORR}=33 \%$ & NR \\
\hline Johnston et al., 2010 [68] & Everolimus, phase II & 19 & 16 & $\mathrm{ORR}=47 \% \mathrm{CR}=5 \%$ & 7.2 months \\
\hline Younes et al., 2012 [72] & Pacritinib, phase I & 34 & 14 & $\mathrm{ORR}=14 \%$ & 130 days \\
\hline Younes et al., 2003 [74] & Rituximab, phase II & 22 & 18 & $\mathrm{ORR}=22 \%$ & 7.8 months \\
\hline Fehniger et al., 2011 [76] & Lenalidomide, phase II & 38 & 33 & $\mathrm{ORR}=17 \%$ & 15 months \\
\hline $\begin{array}{l}\text { Kuruvilla et al., } 2008 \\
\text { [77] }\end{array}$ & Lenalidomide, phase II & 14 & 10 & $\mathrm{PR}=14 \% \mathrm{SD}=50 \%$ & $\begin{array}{l}\text { Median OS was } 9.1 \\
\text { months }\end{array}$ \\
\hline Böll et al., 2010 [78] & Lenalidomide phase II & 42 & NR & $\mathrm{ORR}=50 \%$ & $\mathrm{NR}$ \\
\hline Ansell et al., 2015 [86] & Nivolumab, phase I & 23 & 18 & $\mathrm{ORR}=87 \% \mathrm{SD}=13 \%$ & PFS in 24 weeks was $86 \%$ \\
\hline $\begin{array}{l}\text { Moskowitz et al. } 2014 \\
{[87]}\end{array}$ & Pembrolizumab, phase I & 15 & 15 & $\mathrm{ORR}=53 \% \mathrm{CR}=20 \%$ & NR \\
\hline
\end{tabular}

Pts. N1: total number of patients; Pts. N2: patients who received prior auto-SCT; ORR: overall response rate; CR: complete remission; PR: partial remission; SD: stable disease; OS: overall survival; PFS: progression-free survival; NR: not reported.

four (17\%) had a CR, 16 (70\%) had a PR, and three (13\%) had SD. In 24 weeks, the rate of PFS was $86 \%$ resulting in a very high proportion of patients achieving an overall response and clinical benefit. The study shows promising results; however, larger trials are needed before introducing nivolumab in HL treatment.

A multicenter, open-label, phase Ib clinical trial is ongoing evaluating the use of the humanized IgG4 monoclonal antibody pembrolizumab (formerly MK-3475), targeting the PD-1 receptor, in relapsed or refractory HL patients who failed brentuximab vedotin treatment, with adequate performance status and organ function [87]. Pembrolizumab $10 \mathrm{mg} / \mathrm{kg}$ was administered in 15 patients intravenously every 2 weeks until confirmed tumor progression, excessive toxicity, or completion of 2 years of therapy. The drug was well tolerated with no serious adverse events, and only one patient experienced grade III pain and grade III joint swelling. The most common drug-related adverse events were grade I/II respiratory events $(20 \%)$ and thyroid disorders (20\%). Three patients (20\%) had a CR in 12 weeks. Five additional patients (33\%) had a PR as the best overall response, for an ORR of $53 \%$. Four patients $(27 \%)$ experienced progressive disease, although all 4 experienced a decrease in their overall tumor burden. In conclusion, pembrolizumab therapy appears to be safe, tolerable, and associated with clinical benefit in patients with heavily pretreated HL.

\section{Conclusions}

Auto-SCT is the standard of care for refractory/relapsed HL, leading to long-lasting responses in approximately $50 \%$ of relapsed patients and in a minority of refractory patients. Patients progressing after intensive treatments, such as autoSCT, have a very poor outcome.

In the recent past, particularly effective novel therapies have been identified to treat these patients (Table 1). These agents have all been tested as single drugs acting on different pathways implicated in the pathogenesis of HL (Table 2), and therefore an important future approach will be to combine them with each other and with standard chemotherapies.

Up to now, brentuximab vedotin is the only FDA approved drug for the treatment of relapsed HL. There have been attempts to combine brentuximab vedotin in a pretransplant setting, either in sequential mode, that is, brentuximab vedotin as a single agent, followed by HDC, or concurrently (i.e., with bendamustine). Either way, there is an improvement in the overall response rate and complete response rate with these treatment strategies, and this may evolve with time to include brentuximab vedotin as part of the induction in pretransplant regimens.

PD1-targeted therapies, pembrolizumab and nivolumab, are becoming very good potential drugs, and most likely both will be approved in the near future. 
TABLE 2: Competitive environment.

\begin{tabular}{|c|c|c|c|}
\hline Agent & Indication & Development stage & Mechanism of action \\
\hline Brentuximab vedotin & HL, NHL & Approved for HL and NHL & $\begin{array}{l}\text { Anti-CD30 antibody-drug } \\
\text { conjugate }\end{array}$ \\
\hline Bendamustine & NHL, MM & Approved for NHL & Bifunctional alkylating agent \\
\hline Panobinostat & $\begin{array}{l}\text { AML, CML, breast cancer, prostate } \\
\text { cancer, MM, idiopathic } \\
\text { myelofibrosis, HL, NHL }\end{array}$ & Approved for MM & HDAC inhibitor \\
\hline Mocetinostat & $\begin{array}{c}\text { AML, solid tumors, CLL, MDS, } \\
\text { NHL, HL }\end{array}$ & Phase II & HDAC inhibitor \\
\hline Everolimus & $\begin{array}{c}\text { Solid tumors, transplant rejection, } \\
\text { HL, NHL }\end{array}$ & $\begin{array}{l}\text { Approved for solid tumors and } \\
\text { transplant rejection }\end{array}$ & mTOR inhibitor \\
\hline Pacritinib & $\begin{array}{c}\text { AML, myeloproliferative disorders, } \\
\text { HL, NHL }\end{array}$ & Phase III & JAK2-inhibitor \\
\hline Rituximab & $\begin{array}{c}\text { NHL, CLL, rheumatoid arthritis, } \\
\text { HL, granulomatosis, multiple } \\
\text { sclerosis, MM }\end{array}$ & $\begin{array}{l}\text { Approved for NHL, rheumatoid } \\
\text { arthritis, granulomatosis, CLL }\end{array}$ & Anti-CD20 antibody \\
\hline Lenalidomide & MDS, MM, NHL, HL, CLL & Approved for MDS, MM & Immunomodulator \\
\hline Nivolumab & $\begin{array}{l}\text { Melanoma, lung cancer, renal } \\
\text { cancer, HL }\end{array}$ & Phase I & Anti-PD1 antibody \\
\hline Pembrolizumab & $\begin{array}{l}\text { Melanoma, lung cancer, renal } \\
\text { cancer, HL }\end{array}$ & Phase I & Anti-PD1 antibody \\
\hline
\end{tabular}

HL: Hodgkin lymphoma; NHL: non-Hodgkin lymphoma; MM: multiple myeloma; AML: acute myeloid leukemia; CML: chronic myeloid leukemia; MDS: myelodysplastic syndrome; CLL: chronic lymphatic leukemia.

Although these new therapies have clearly demonstrated efficacy in HL, a molecularly targeted drug achieving longterm responses with good tolerability is still lacking. Moreover, the majority of patients are young, and in this scenario we believe that allo-SCT can play an important role in selected patients.

\section{Conflict of Interests}

The authors report no conflict of interests or funding sources.

\section{Authors' Contribution}

Quality control of data and algorithms has been done by Roberta Fedele, Massimo Martino, Anna Grazia Recchia, Massimo Gentile, and Fortunato Morabito. Paper preparation has been done by Roberta Fedele and Massimo Martino. Paper editing has been done by Roberta Fedele, Massimo Martino, and Anna Grazia Recchia. Paper review has been done by Roberta Fedele, Massimo Martino, Anna Grazia Recchia, and Fortunato Morabito. Approval of the submitted and final versions has been done by Roberta Fedele, Massimo Martino, Anna Grazia Recchia, Massimo Gentile, and Fortunato Morabito. Giuseppe Irrera contributed to paper review and the approval of the submitted and final versions.

\section{References}

[1] R. Siegel, J. Ma, Z. Zou, and A. Jemal, "Cancer statistics, 2014," CA: A Cancer Journal for Clinicians, vol. 64, no. 1, pp. 9-29, 2014.
[2] T. Chisesi, M. Bellei, S. Luminari et al., "Long-term followup analysis of HD9601 trial comparing ABVD versus Stanford $\mathrm{V}$ versus MOPP/EBV/CAD in patients with newly diagnosed advanced-stage Hodgkin's lymphoma: a study from the Intergruppo Italiano Linfomi," Journal of Clinical Oncology, vol. 29, no. 32, pp. 4227-4233, 2011.

[3] T. Moscato, R. Fedele, G. Messina, G. Irrera, G. Console, and M. Martino, "Hematopoietic progenitor cells transplantation for recurrent or refractory Hodgkin's lymphoma," Expert Opinion on Biological Therapy, vol. 13, no. 7, pp. 1013-1027, 2013.

[4] F. Montanari and C. Diefenbach, "Relapsed Hodgkin lymphoma: management strategies," Current Hematologic Malignancy Reports, vol. 9, no. 3, pp. 284-293, 2014.

[5] S. Horning and M. S. Fanale, "Defining a population of Hodgkin lymphoma patients for novel therapeutics: an international effort," Annals of Oncology, vol. 20, article 118, 2008.

[6] D. Buglio, G. Georgakis, and A. Younes, "Novel small-molecule therapy of Hodgkin lymphoma," Expert Review of Anticancer Therapy, vol. 7, no. 5, pp. 735-740, 2007.

[7] R. Küppers, “The biology of Hodgkin's lymphoma," Nature Reviews Cancer, vol. 9, no. 1, pp. 15-27, 2008.

[8] D. E. Reece, M. J. Barnett, J. M. Connors et al., "Intensive chemotherapy with cyclophosphamide, carmustine, and etoposide followed by autologous bone marrow transplantation for relapsed Hodgkin's disease," The Journal of Clinical Oncology, vol. 9, no. 10, pp. 1870-1879, 1991.

[9] R. Chopra, A. K. McMillan, D. C. Linch et al., "The place of high-dose BEAM therapy and autologous bone marrow transplantation in poor-risk Hodgkin's disease. A single-center eightyear study of 155 patients," Blood, vol. 81, no. 5, pp. 1137-1145, 1993. 
[10] A. Sureda, R. Arranz, A. Iriondo et al., "Autologous stem-cell transplantation for Hodgkin's disease: results and prognostic factors in 494 patients from the Grupo Español de Linfomas/ Transplante Autólogo de Médula Ósea Spanish Cooperative Group," Journal of Clinical Oncology, vol. 19, no. 5, pp. 13951404, 2001.

[11] M. D. Caballero, V. Rubio, J. Rifon et al., "BEAM chemotherapy followed by autologous stem cell support in lymphoma patients: analysis of efficacy, toxicity and prognostic factors," Bone Marrow Transplantation, vol. 20, no. 6, pp. 451-458, 1997.

[12] D. C. Linch, D. Winfield, A. H. Goldstone et al., "Dose intensification with autologous bone-marrow transplantation in relapsed and resistant Hodgkin's disease: results of a BNLI randomised trial," The Lancet, vol. 341, no. 8852, pp. 1051-1054, 1993.

[13] N. Schmitz, B. Pfistner, M. Sextro et al., "Aggressive conventional chemotherapy compared with high-dose chemotherapy with autologous haemopoietic stem-cell transplantation for relapsed chemosensitive Hodgkin's disease: a randomised trial," The Lancet, vol. 359, no. 9323, pp. 2065-2071, 2002.

[14] A. Josting, H. Müller, P. Borchmann et al., "Dose intensity of chemotherapy in patients with relapsed Hodgkin's lymphoma," Journal of Clinical Oncology, vol. 28, no. 34, pp. 5074-5080, 2010.

[15] M. Musso, R. Scalone, G. Marcacci et al., "Fotemustine plus etoposide, cytarabine and melphalan (FEAM) as a new conditioning regimen for lymphoma patients undergoing auto-SCT: a multicenter feasibility study," Bone Marrow Transplantation, vol. 45, no. 7, pp. 1147-1153, 2010.

[16] G. Visani, L. Malerba, P. M. Stefani et al., "BeEAM (bendamustine, etoposide, cytarabine, melphalan) before autologous stem cell transplantation is safe and effective for resistant/relapsed lymphoma patients," Blood, vol. 118, no. 12, pp. 3419-3425, 2011.

[17] R. L. Tombleson, M. R. Green, and K. M. Fancher, "Putting caution in TEAM: high-dose chemotherapy with autologous HSCT for primary central nervous system lymphoma," Bone Marrow Transplantation, vol. 47, no. 10, pp. 1383-1384, 2012.

[18] J. W. Sweetenham, A. M. Carella, G. Taghipour et al., "Highdose therapy and autologous stem-cell transplantation for adult patients with Hodgkin's disease who do not enter remission after induction chemotherapy: results in 175 patients reported to the European group for blood and marrow transplantation," Journal of Clinical Oncology, vol. 17, no. 10, pp. 3101-3109, 1999.

[19] H. M. Lazarus, P. A. Rowlings, M.-J. Zhang et al., "Autotransplants for Hodgkin's disease in patients never achieving remission: a report from the autologous blood and marrow transplant registry," Journal of Clinical Oncology, vol. 17, no. 2, pp. 534-545, 1999.

[20] M. Constans, A. Sureda, M. J. Terol et al., "Autologous stem cell transplantation for primary refractory Hodgkin's disease: results and clinical variables affecting outcome," Annals of Oncology, vol. 14, no. 5, pp. 745-751, 2003.

[21] T. Ahmed, K. Rashid, F. Waheed et al., "Long-term survival of patients with resistant lymphoma treated with tandem stem cell transplant," Leukemia and Lymphoma, vol. 46, no. 3, pp. 405414, 2005.

[22] H. C. Fung, P. Stiff, J. Schriber et al., “Tandem autologous stem cell transplantation for patients with primary refractory or poor risk recurrent Hodgkin lymphoma," Biology of Blood and Marrow Transplantation, vol. 13, no. 5, pp. 594-600, 2007.

[23] L. Castagna, M. Magagnoli, M. Balzarotti et al., "Tandem highdose chemotherapy and autologous stem cell transplantation in refractory/relapsed Hodgkin's lymphoma: a monocenter prospective study," American Journal of Hematology, vol. 82, no. 2, pp. 122-127, 2007.

[24] F. Morschhauser, P. Brice, C. Fermé et al., "Risk-adapted salvage treatment with single or tandem autologous stem-cell transplantation for first relapse/refractory Hodgkin's lymphoma: results of the prospective multicenter $\mathrm{H} 96$ trial by the GELA/ SFGM study group," Journal of Clinical Oncology, vol. 26, no. 36, pp. 5980-5987, 2008.

[25] M. A. Perales, I. Ceberio, P. Armand et al., "Role of cytotoxic therapy with hematopoietic cell transplantation in the treatment of Hodgkin lymphoma: guidelines from the American Society for Blood and Marrow Transplantation," Biology of Blood and Marrow Transplantation, vol. 21, no. 6, pp. 971-983, 2015.

[26] K. J. Thomson, K. S. Peggs, P. Smith et al., "Superiority of reduced-intensity allogeneic transplantation over conventional treatment for relapse of Hodgkin's lymphoma following autologous stem cell transplantation," Bone Marrow Transplantation, vol. 41, no. 9, pp. 765-770, 2008.

[27] L. Castagna, B. Sarina, E. Todisco et al., "Allogeneic stem cell transplantation compared with chemotherapy for poor-risk Hodgkin lymphoma," Biology of Blood and Marrow Transplantation, vol. 15, no. 4, pp. 432-438, 2009.

[28] B. Sarina, L. Castagna, L. Farina et al., "Allogeneic transplantation improves the overall and progression-free survival of Hodgkin lymphoma patients relapsing after autologous transplantation: a retrospective study based on the time of HLA typing and donor availability," Blood, vol. 115, no. 18, pp. 3671-3677, 2010.

[29] J. L. Gajewski, G. L. Phillips, K. A. Sobocinski et al., "Bone marrow transplants from HLA-identical siblings in advanced Hodgkin's disease," Journal of Clinical Oncology, vol. 14, no. 2, pp. 572-578, 1996.

[30] A. Claviez, C. Canals, D. Dierickx et al., "Allogeneic hematopoietic stem cell transplantation in children and adolescents with recurrent and refractory Hodgkin lymphoma: an analysis of the European Group for Blood and Marrow Transplantation," Blood, vol. 114, no. 10, pp. 2060-2067, 2009.

[31] K. S. Peggs, I. Kayani, N. Edwards et al., "Donor lymphocyte infusions modulate relapse risk in mixed chimeras and induce durable salvage in relapsed patients after T-cell-depleted allogeneic transplantation for Hodgkin's lymphoma," Journal of Clinical Oncology, vol. 29, no. 8, pp. 971-978, 2011.

[32] N. Milpied, A. K. Fielding, R. M. Pearce, P. Ernst, and A. H. Goldstone, "Allogeneic bone marrow transplant is not better than autologous transplant for patients with relapsed Hodgkin's disease," Journal of Clinical Oncology, vol. 14, no. 4, pp. 12911296, 1996.

[33] J. E. Anderson, M. R. Litzow, F. R. Appelbaum et al., "Allogeneic, syngeneic, and autologous marrow transplantation for Hodgkin's disease: the 21-year seattle experience," Journal of Clinical Oncology, vol. 11, no. 12, pp. 2342-2350, 1993.

[34] A. Sureda, S. Robinson, C. Canals et al., "Reduced-intensity conditioning compared with conventional allogeneic stem-cell transplantation in relapsed or refractory Hodgkin's lymphoma: an analysis from the lymphoma working party of the European Group for Blood and Marrow Transplantation," Journal of Clinical Oncology, vol. 26, no. 3, pp. 455-462, 2008.

[35] J. R. Passweg, H. Baldomero, A. Gratwohl et al., “The EBMT activity survey: 1990-2010," Bone Marrow Transplantation, vol. 47, no. 7, pp. 906-923, 2012. 
[36] S. P. Robinson, A. Sureda, C. Canals et al., "Reduced intensity conditioning allogeneic stem cell transplantation for Hodgkin's lymphoma: identification of prognostic factors predicting outcome," Haematologica, vol. 94, no. 2, pp. 230-238, 2009.

[37] L. M. Burroughs, P. V. O’Donnell, B. M. Sandmaier et al., "Comparison of outcomes of HLA-matched related, unrelated, or HLA-haploidentical related hematopoietic cell transplantation following nonmyeloablative conditioning for relapsed or refractory Hodgkin lymphoma," Biology of Blood and Marrow Transplantation, vol. 14, no. 11, pp. 1279-1287, 2008.

[38] A. Raiola, A. Dominietto, R. Varaldo et al., "Unmanipulated haploidentical BMT following non-myeloablative conditioning and post-transplantation CY for advanced Hodgkin's lymphoma," Bone Marrow Transplantation, vol. 49, no. 2, pp. 190194, 2014.

[39] L. Luznik, P. V. O’Donnell, H. J. Symons et al., "HLAhaploidentical bone marrow transplantation for hematologic malignancies using nonmyeloablative conditioning and highdose, posttransplantation cyclophosphamide," Biology of Blood and Marrow Transplantation, vol. 14, no. 6, pp. 641-650, 2008.

[40] A. Sureda, C. Canals, R. Arranz et al., "Allogeneic stem cell transplantation after reduced intensity conditioning in patients with relapsed or refractory Hodgkin's lymphoma. Results of the HDR-ALLO study-a prospective clinical trial by the Grupo Español de Linfomas/Trasplante de Médula Osea (GEL/ TAMO) and the Lymphoma Working Party of the European Group for Blood and Marrow Transplantation," Haematologica, vol. 97, no. 2, pp. 310-317, 2012.

[41] K. Horstmann, A. Boumendil, J. Finke et al., "Second alloSCT in patients with lymphoma relapse after a first allogeneic transplantation. A retrospective study of the EBMT Lymphoma Working Party," Bone Marrow Transplantation, vol. 50, no. 6, pp. 790-794, 2015.

[42] C. Kahl, B. E. Storer, B. M. Sandmaier et al., "Relapse risk in patients with malignant diseases given allogeneic hematopoietic cell transplantation after nonmyeloablative conditioning," Blood, vol. 110, no. 7, pp. 2744-2748, 2007.

[43] A. Younes and A. Carbone, "CD30/CD30 ligand and CD40/ CD40 ligand in malignant lymphoid disorders," The International Journal of Biological Markers, vol. 14, no. 3, pp. 135-143, 1999.

[44] A. Younes and B. B. Aggarwall, "Clinical implications of the tumor necrosis factor family in benign and malignant hematologic disorders," Cancer, vol. 98, no. 3, pp. 458-467, 2003.

[45] S. M. Ansell, S. M. Horwitz, A. Engert et al., "Phase I/II study of an anti-CD30 monoclonal antibody (MDX-060) in Hodgkin's lymphoma and anaplastic large-cell lymphoma," Journal of Clinical Oncology, vol. 25, no. 19, pp. 2764-2769, 2007.

[46] A. Forero-Torres, J. P. Leonard, A. Younes et al., "A Phase II study of SGN-30 (anti-CD30 mAb) in Hodgkin lymphoma or systemic anaplastic large cell lymphoma," British Journal of Haematology, vol. 146, no. 2, pp. 171-179, 2009.

[47] E. Oflazoglu, K. M. Kissler, E. L. Sievers, I. S. Grewal, and H.-P. Gerber, "Combination of the anti-CD30-auristatin-E antibodydrug conjugate (SGN-35) with chemotherapy improves antitumour activity in Hodgkin lymphoma," British Journal of Haematology, vol. 142, no. 1, pp. 69-73, 2008.

[48] A. Younes, N. L. Bartlett, J. P. Leonard et al., "Brentuximab vedotin (SGN-35) for relapsed CD30-positive lymphomas," The New England Journal of Medicine, vol. 363, no. 19, pp. 1812-1821, 2010.
[49] A. Younes, A. K. Gopal, S. E. Smith et al., "Results of a pivotal phase II study of brentuximab vedotin for patients with relapsed or refractory Hodgkin's lymphoma," Journal of Clinical Oncology, vol. 30, no. 18, pp. 2183-2189, 2012.

[50] A. K. Gopal, R. Chen, S. E. Smith et al., "Durable remissions in a pivotal phase 2 study of brentuximab vedotin in relapsed or refractory Hodgkin lymphoma," Blood, vol. 125, no. 8, pp. 12361243, 2015.

[51] C. H. Moskowitz, A. Nademanee, T. Masszi et al., "Brentuximab vedotin as consolidation therapy after autologous stem-cell transplantation in patients with Hodgkin's lymphoma at risk of relapse or progression (AETHERA): a randomised, doubleblind, placebo-controlled, phase 3 trial," The Lancet, vol. 385, no. 9980, pp. 1853-1862, 2015.

[52] P. L. Zinzani, P. Corradini, A. M. Gianni et al., "Brentuximab vedotin in CD30-positive lymphomas. A SIE, SIES, GITMO position paper," Clinical Lymphoma, Myeloma \& Leukemia, vol. 15, no. 9, pp. 507-513, 2015.

[53] V. Gandhi, "Metabolism and mechanisms of action of bendamustine: rationales for combination therapies," Seminars in Oncology, vol. 4, supplement 13, pp. 4-11, 2002.

[54] M. M. Goldenberg, "Pharmaceutical approval update," $P \& T$, vol. 33, no. 5, pp. 299-302, 2008.

[55] A. J. Moskowitz, P. A. Hamlin Jr., M.-A. Perales et al., "Phase II study of bendamustine in relapsed and refractory Hodgkin lymphoma," Journal of Clinical Oncology, vol. 31, no. 4, pp. 456460, 2013.

[56] P. L. Zinzani, E. Derenzini, C. Pellegrini, M. Celli, A. Broccoli, and L. Argnani, "Bendamustine efficacy in Hodgkin lymphoma patients relapsed/refractory to brentuximab vedotin," British Journal of Haematology, vol. 163, no. 5, pp. 681-683, 2013.

[57] P. L. Zinzani, U. Vitolo, S. Viviani et al., "Safety and efficacy of single-agent bendamustine after failure of brentuximab vedotin in patients with relapsed or refractory Hodgkin's lymphoma: experience with 27 patients," Clinical Lymphoma, Myeloma and Leukemia, vol. 15, no. 7, pp. 404-408, 2015.

[58] A. LaCasce, R. G. Bociek, J. Matous et al., "Brentuximab vedotin in combination with bendamustine for patients with Hodgkin lymphoma who are relapsed or refractory after frontline therapy," Blood, vol. 124, no. 21, p. 293, 2014.

[59] O. A. O'Connor, J. Kuruvilla, A. Sawas et al., "A Phase 1-2 study of brentuximab vedotin $(\mathrm{Bv})$ and bendamustine $(\mathrm{B})$ in patients with relapsed or refractory hodgkin lymphoma (HL) and anaplastic large T-cell lymphoma (ALCL)," Blood, vol. 124, no. 21, p. 3084, 2014.

[60] M. Dickinson, D. Ritchie, D. J. Deangelo et al., "Preliminary evidence of disease response to the pan deacetylase inhibitor panobinostat (LBH589) in refractory Hodgkin Lymphoma," British Journal of Haematology, vol. 147, no. 1, pp. 97-101, 2009.

[61] A. Younes, A. Sureda, D. Ben-Yehuda et al., "Panobinostat in patients with relapsed/refractory Hodgkin's lymphoma after autologous stem-cell transplantation: results of a phase II study," Journal of Clinical Oncology, vol. 30, no. 18, pp. 2197-2203, 2012.

[62] P. Atadja, "Development of the pan-DAC inhibitor panobinostat (LBH589): successes and challenges," Cancer Letters, vol. 280, no. 2, pp. 233-241, 2009.

[63] M. Lemoine, E. Derenzini, D. Buglio et al., “The pandeacetylase inhibitor panobinostat induces cell death and synergizes with everolimus in Hodgkin lymphoma cell lines," Blood, vol. 119, no. 17, pp. 4017-4025, 2012.

[64] B. Christian, A. Kopko, T. A. Fehniger, N. L. Bartlett, and K. A. A. Blum, "Phase I. Trial of the histone deacetylase (HDAC) 
inhibitor, panobinostat, in combination with lenalidomide in patients with relapsed/refractory Hodgkin's lymphoma (HL)," Blood, vol. 120, no. 21, p. 1644, 2012.

[65] A. Younes, Y. Oki, R. G. Bociek et al., "Mocetinostat for relapsed classical Hodgkin's lymphoma: an open-label, single-arm, phase 2 trial," The Lancet Oncology, vol. 12, no. 13, pp. 1222-1228, 2011.

[66] N. T. Ihle and G. Powis, "Take your PIK: phosphatidylinositol 3-kinase inhibitors race through the clinic and toward cancer therapy," Molecular Cancer Therapeutics, vol. 8, no. 1, pp. 1-9, 2009.

[67] A. Guarini, C. Minoia, M. Giannoccaro et al., "mTOR as a target of everolimus in refractory/relapsed Hodgkin Lymphoma," Current Medicinal Chemistry, vol. 19, no. 7, pp. 945-954, 2012.

[68] P. B. Johnston, D. J. Inwards, J. P. Colgan et al., "A phase II trial of the oral mTOR inhibitor everolimus in relapsed Hodgkin lymphoma," American Journal of Hematology, vol. 85, no. 5, pp. 320-324, 2010.

[69] A. Carbone, A. Gloghini, L. Castagna, A. Santoro, and C. Carlo-Stella, "Primary refractory and early-relapsed Hodgkin's lymphoma: strategies for therapeutic targeting based on the tumour microenvironment," The Journal of Pathology, vol. 237, no. 1, pp. 4-13, 2015.

[70] J. Munoz, N. Dhillon, F. Janku, S. S. Watowich, and D. S. Hong, "STAT3 inhibitors: finding a home in lymphoma and leukemia," The Oncologist, vol. 19, no. 5, pp. 536-544, 2014.

[71] L. M. Scott and M. K. Gandhi, "Deregulated JAK/STAT signalling in lymphomagenesis, and its implications for the development of new targeted therapies," Blood Reviews, 2015.

[72] A. Younes, J. Romaguera, M. Fanale et al., "Phase I study of a novel oral Janus kinase 2 inhibitor, SB1518, in patients with relapsed lymphoma: evidence of clinical and biologic activity in multiple lymphoma subtypes," Journal of Clinical Oncology, vol. 30, no. 33, pp. 4161-4167, 2012.

[73] R. J. Jones, C. D. Gocke, Y. L. Kasamon et al., "Circulating clonotypic B cells in classic Hodgkin lymphoma," Blood, vol. 113, no. 23, pp. 5920-5926, 2009.

[74] A. Younes, J. Romaguera, F. Hagemeister et al., "A pilot study of rituximab in patients with recurrent, classic Hodgkin disease," Cancer, vol. 98, no. 2, pp. 310-314, 2003.

[75] R. Ramchandren, "Advances in the treatment of relapsed or refractory Hodgkin's lymphoma," The Oncologist, vol. 17, no. 3, pp. 367-376, 2012.

[76] T. A. Fehniger, S. Larson, K. Trinkaus et al., "A phase 2 multicenter study of lenalidomide in relapsed or refractory classical Hodgkin lymphoma," Blood, vol. 118, no. 19, pp. 51195125, 2011.

[77] J. Kuruvilla, D. Taylor, L. Wang, C. Blattler, A. Keating, and M. Crump, "Phase II trial of Lenalidomide in patients with relapsed or refractory Hodgkin lymphoma," Blood, vol. 112, no. 11, p. 3052, 2008.

[78] B. Böll, M. Fuchs, K. S. Reiners et al., "Lenalidomide in patients with relapsed or refractory Hodgkin lymphoma," Blood, vol. 116, no. 21, p. 2828, 2010.

[79] B. Böll, A. Plutschow, M. Fuchs et al., "German hodgkin study group phase I trial of doxorubicin, vinblastine, dacarbazine, and lenalidomide (AVD-Rev) for older Hodgkin lymphoma patients," Blood, vol. 122, no. 21, p. 3054, 2013.

[80] D. M. Pardoll, "The blockade of immune checkpoints in cancer immunotherapy," Nature Reviews Cancer, vol. 12, no. 4, pp. 252$264,2012$.
[81] S. Topalian, C. Drake, and D. Pardoll, "Immune checkpoint blockade: a common denominator approach to cancer therapy," Cancer Cell, vol. 27, no. 4, pp. 450-461, 2015.

[82] A. Ribas, "Tumor immunotherapy directed at PD-1," The New England Journal of Medicine, vol. 366, no. 26, pp. 2517-2519, 2012.

[83] S. L. Topalian, F. S. Hodi, J. R. Brahmer et al., "Safety, activity, and immune correlates of anti-PD-1 antibody in cancer," The New England Journal of Medicine, vol. 366, no. 26, pp. 24432454, 2012

[84] M. A. Postow, M. K. Callahan, and J. D. Wolchok, "Immune checkpoint blockade in cancer therapy," Journal of Clinical Oncology, vol. 33, no. 17, pp. 1974-1982, 2015.

[85] M. R. Green, S. Monti, S. J. Rodig et al., "Integrative analysis reveals selective 9p24.1 amplification, increased PD-1 ligand expression, and further induction via JAK2 in nodular sclerosing Hodgkin lymphoma and primary mediastinal large B-cell lymphoma," Blood, vol. 116, no. 17, pp. 3268-3277, 2010.

[86] S. M. Ansell, A. M. Lesokhin, I. Borrello et al., "PD-1 blockade with nivolumab in relapsed or refractory Hodgkin's lymphoma," The New England Journal of Medicine, vol. 372, no. 4, pp. 311-319, 2015.

[87] C. H. Moskowitz, V. Ribrag, J. M. Michot et al., "PD-1 Blockade with the monoclonal antibody Pembrolizumab (MK-3475) in patients with classical Hodgkin lymphoma after brentuximab vedotin failure: preliminary results from a Phase $1 \mathrm{~B}$ study (KEYNOTE-013)," Blood, vol. 124, no. 21, p. 290, 2014. 


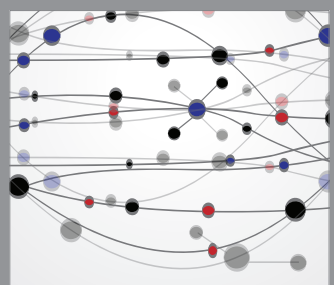

The Scientific World Journal
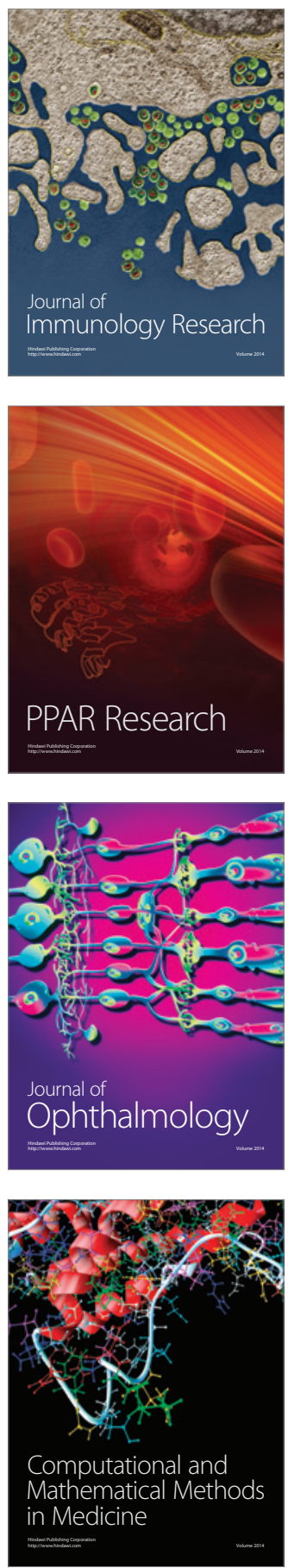

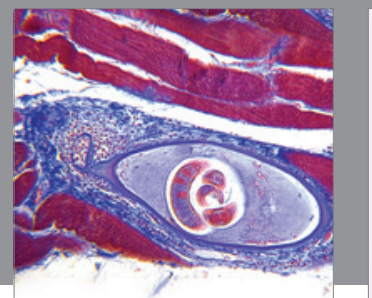

Gastroenterology

Research and Practice
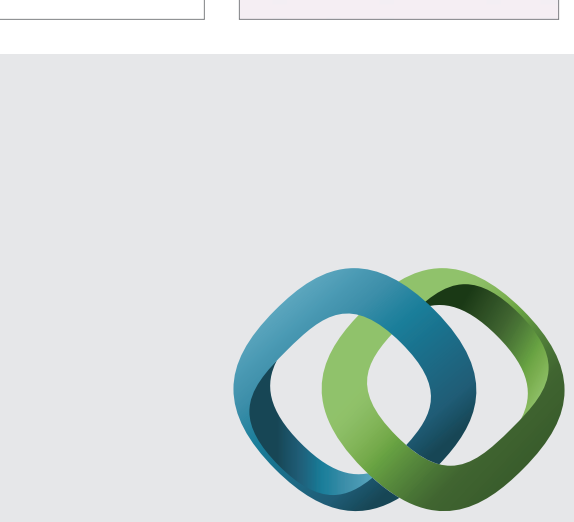

\section{Hindawi}

Submit your manuscripts at

http://www.hindawi.com
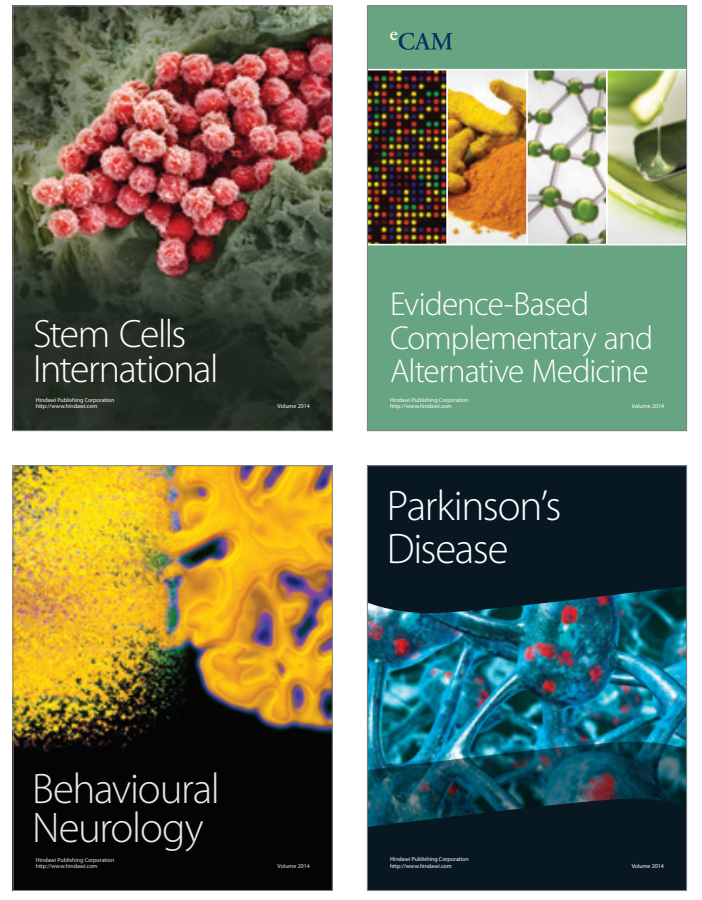
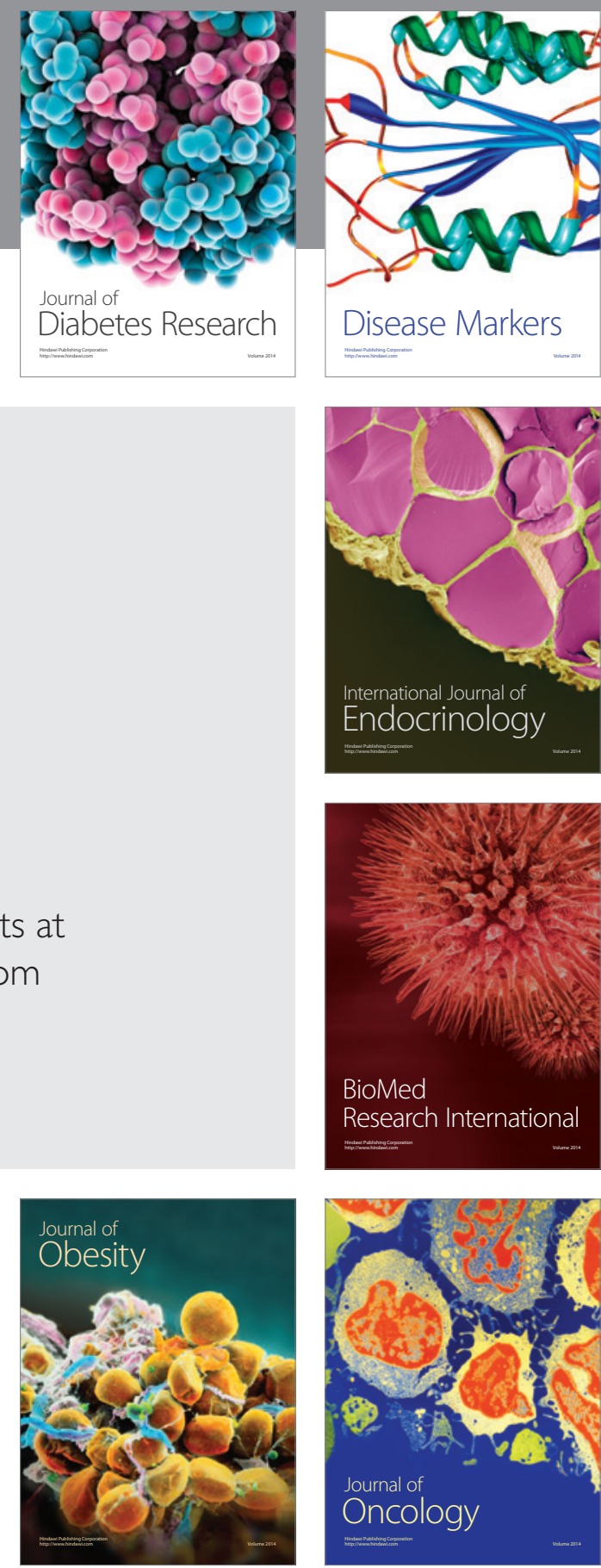

Disease Markers
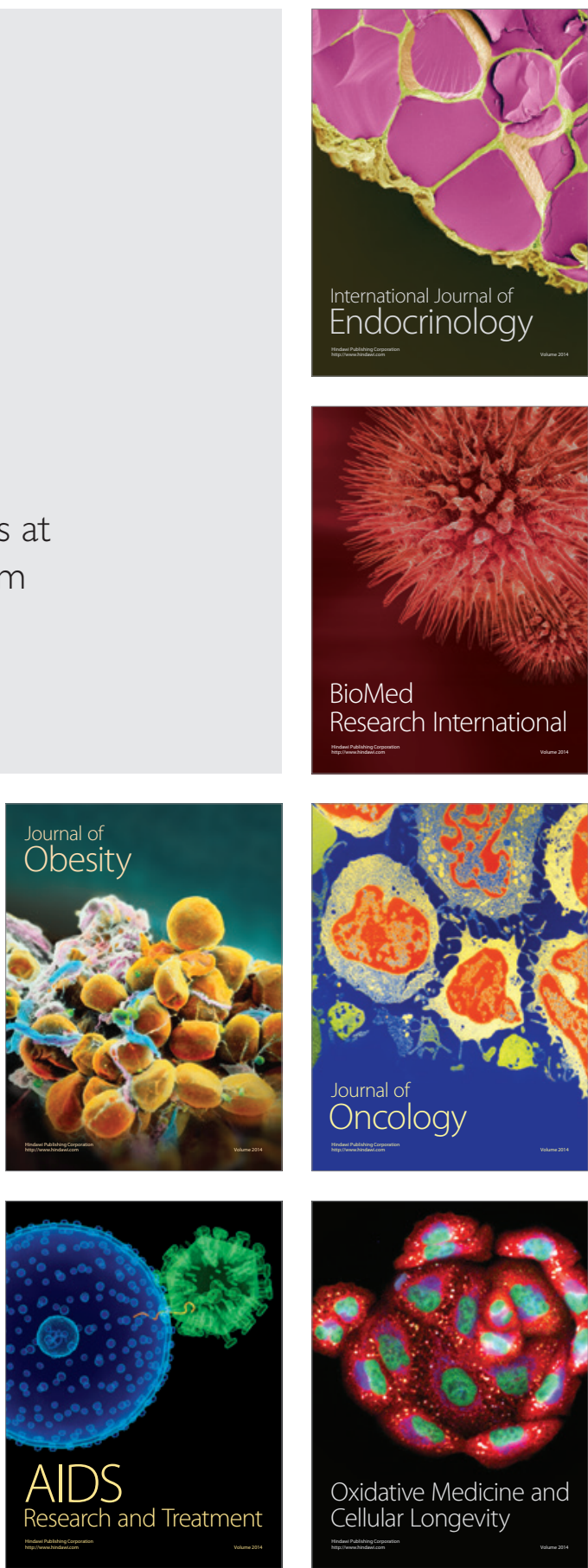